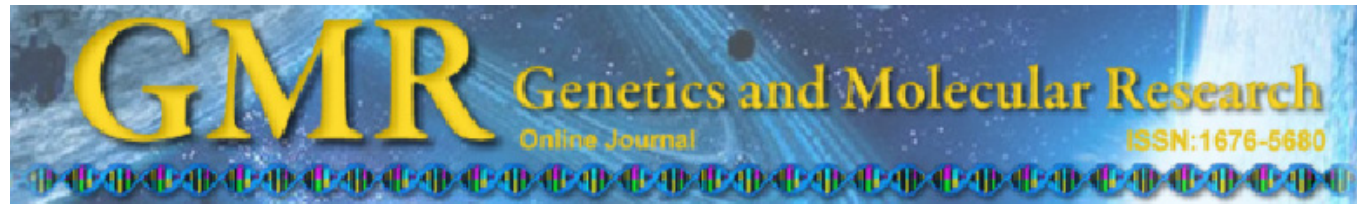

$\underline{\text { Case Report }}$

\title{
Clonal monosomy of chromosome 21 in a case of myelodysplastic syndrome
}

\author{
P.C. Freitas ${ }^{1}$, A.B. Carvalho-Salles ${ }^{2}$, C.F. Mendiburu ${ }^{1}$, O. Ricci Jr. ${ }^{3}$ and \\ A.C. Fett-Conte ${ }^{2}$ \\ ${ }^{1}$ Departamento de Biologia, Instituto de Biociências, Letras e Ciências Exatas, \\ Universidade Estadual de São Paulo "Julio de Mesquita Filho", \\ São José do Rio Preto, SP, Brasil \\ ${ }^{2}$ Departamento de Biologia Molecular, Escola de Medicina de São José do Rio Preto, \\ São José do Rio Preto, SP, Brasil \\ ${ }^{3}$ Departamento de Medicina II, Escola de Medicina de São José do Rio Preto, \\ São José do Rio Preto, SP, Brasil \\ Corresponding author: A.C. Fett-Conte \\ E-mail: genetica@famerp.br
}

Genet. Mol. Res. 10 (4): 2718-2720 (2011)

Received December 2, 2010

Accepted July 3, 2011

Published November 4, 2011

DOI http://dx.doi.org/10.4238/2011.November.4.5

\begin{abstract}
This study reports on a cytogenetic finding in a bone marrow examination of a 47-year-old male patient treated in the Hematology and Blood Transfusion Service of the Hospital de Base in São José do Rio Preto, São Paulo State, Brazil. The only alteration found at diagnosis of myelodysplastic syndrome (MDS) subtype refractory anemia with excess blasts (RAEB-2) was clonal monosomy of chromosome 21. The patient evolved to acute myeloid leukemia type M2 and died nine months after diagnosis. Clonal monosomy of chromosome 21, as the only cytogenetic abnormality in MDS, has only been reported three times previously. This uncommon cytogenetic abnormality in MDS has been associated with a poor clinical course, although more data will be needed to determine if this prognosis is invariable.
\end{abstract}

Key words: Myelodysplastic syndrome; Monosomy 21;

Clonal chromosomal abnormalities 
Myelodysplastic syndromes (MDS) are clonal stem cell disorders characterized by ineffective hematopoiesis leading to bone marrow failure, peripheral blood cytopenias, dysplasia, and a high risk of progression to acute leukemia (Wang et al., 2010). Complications from cytopenias lead to higher morbidity and mortality among patients with MDS compared to an age-matched population. The variable risk of progression to acute myeloid leukemia (AML) is of major prognostic significance in early MDS without blast excess (Haase et al., 2007). MDS is generally classified as "primary" (or de novo) or "treatment-related" (secondary to cytotoxic chemotherapy) (Malcovati and Nimer, 2008). Onset of disease in the majority of patients occurs in the seventh decade of life (Mufti et al., 2008). According to the WHO classification of MDS (2008), refractory anemia with excess blast (RAEB) includes patients with 5-19\% blasts in the bone marrow (Jadersten and Hellstrom-Lindberg, 2009).

Chromosomal abnormalities are major determinants in the pathogenesis, diagnosis, and prognosis of MDS and are increasingly the basis for selecting drugs for individual patients. Cytogenetic analysis of the bone marrow is indicated in MDS not only to detect characteristic chromosomal abnormalities, but also to assess clonal evolution (Haase et al., 2007).

Most frequently, a loss of genetic material in the form of deletions and monosomies can be observed, although translocations with loss or gain can also occur (Haase, 2008; Malcovati and Nimer, 2008). Due to genetic heterogeneity, knowledge about distinct cytogenetic alterations is mainly restricted to the most common abnormalities $(-5 / 5 q-,-7 / 7 q-,+8,20 q-$, and -Y). However, in MDS, rare chromosome abnormalities are present in a substantial portion of patients. In a recent review study of the German-Austrian MDS Study Group, 59\% of 2370 abnormalities observed in 1080 patients with MDS were uncommon. Monosomy of chromosome 21 as the only cytogenetic abnormality in MDS was observed in three cases so it is therefore a very rare alteration. There was no mention of the prognosis in these cases. An association of monosomy of chromosome 21 with other cytogenetic alterations was described in 30 cases with prognosis being reported as favorable with a median survival of 32 months. However, this was true only when not more than one additional abnormality was present (Haase, 2008).

Our study was approved by the Ethics Committee of FAMERP. We describe a patient with RAEB- 2 who presented monosomy 21 as the only clonal alteration in bone marrow cells.

The 47-year-old man was seen in April 2008. According to the patient, when he had been treated in another hospital six years previously, he was diagnosed as having undifferentiated gastric carcinoma and large cell lymphoma. Total gastrectomy was performed and the patient was submitted to chemotherapy for one year. His whole blood count showed pancytopenia with hemoglobin at $6.8 \mathrm{~g} / \mathrm{dL}$, hematocrit at $28 \%$, a white blood cell count of $2700 / \mu \mathrm{L}$, and platelets at $2300 / \mu \mathrm{L}$. The myelogram showed $18 \%$ of blasts and a cytochemical test was Sudan-Black positive. These findings were consistent with the diagnosis of RAEB-2.

A bone marrow aspirate drawn at this diagnosis was used for cytogenetic analysis after a 24-h non-stimulated culture in RPMI-1640 medium with $20 \%$ fetal calf serum. GTGbanding karyotype was described according to the International System for Human Cytogenetic Nomenclature (Shaffer and Tommerup, 2009). Of the 17 metaphases analyzed from different culture flasks, $10(59 \%)$ demonstrated monosomy of chromosome $21(45, \mathrm{XY},-21)$ and 7 were normal $(46, \mathrm{XY})$. Figure 1 illustrates three metaphases with monosomy of chromosome 21 ; this was the only clonal chromosomal abnormality found.

Two months later, in June 2008, the patient presented AML type M2 with the bone marrow showing $54 \%$ blasts and a high nucleus/cytoplasm ratio, evident nucleoli and positive 
peroxidase. Immunophenotyping showed an immunophenotypic profile of CD45+, CD34+, CD33+, CD117+, CD13+, HLADR+, CD14+, CD15+, CD4+, CD54+, and CD42a-consistent with a diagnosis of AML (M2). He began chemotherapy using a doxorubicin and cytarabine protocol $(7+3)$. The patient died in January 2009 due to a stroke nine months after the diagnosis of RAEB-2.
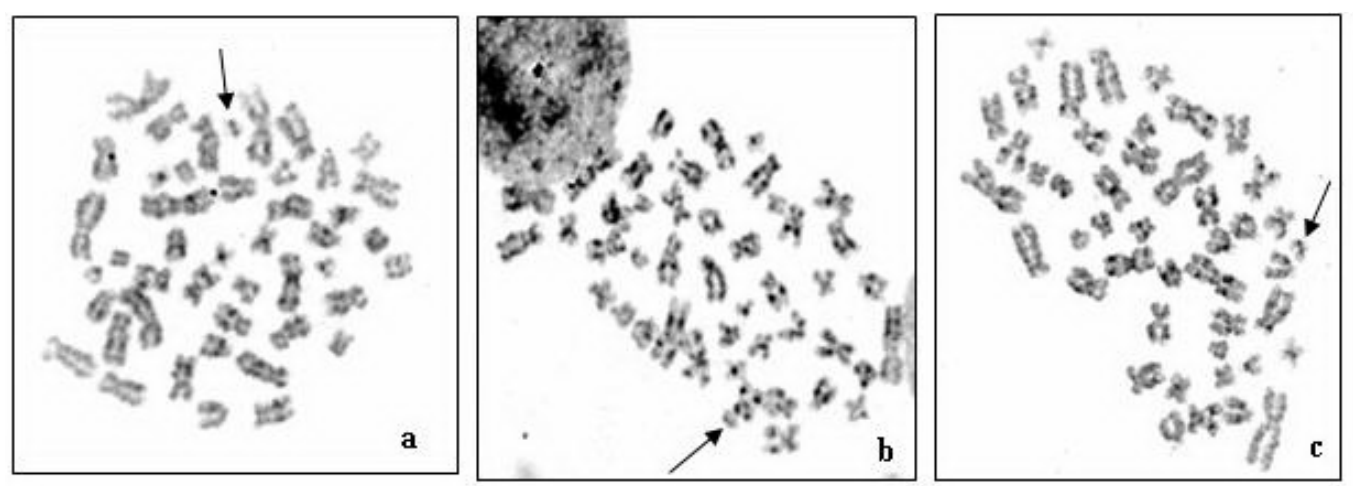

Figure 1. a. b. c. Metaphases of a patient showing monosomy of chromosome 21 (arrows).

The presence of monosomy of chromosome 21 as the sole clonal cytogenetic abnormality in malignant cells seems to be related to an unfavorable prognosis in secondary MDS. Its role in the development of the disease should be further studied with detection being important for prognosis and therapeutic management.

\section{ACKNOWLEDGMENTS}

The authors wish to thank the parents and clinician for the data. Research supported by the Higher Education Professional Training Board (CAPES), Brazil.

\section{REFERENCES}

Haase D (2008). Cytogenetic features in myelodysplastic syndromes. Ann. Hematol. 87: 515-526.

Haase D, Germing U, Schanz J, Pfeilstocker M, et al. (2007). New insights into the prognostic impact of the karyotype in MDS and correlation with subtypes: evidence from a core dataset of 2124 patients. Blood 110: 4385-4395.

Jadersten M and Hellstrom-Lindberg E (2009). Myelodysplastic syndromes: biology and treatment. J. Intern. Med. 265: 307-328.

Malcovati L and Nimer SD (2008). Myelodysplastic syndromes: diagnosis and staging. Cancer Control 15: 4-13.

Mufti GJ, Bennett JM, Goasguen J, Bain BJ, et al. (2008). Diagnosis and classification of myelodysplastic syndrome: international working group on morphology of myelodysplastic syndrome (IWGM-MDS) consensus proposals for the definition and enumeration of myeloblasts and ring sideroblasts. Haematologica 93: 1712-1717.

Shaffer LG and Tommerup N (2009). An International System for Human Cytogenetic Nomenclature ISCN. ND-S Karger Publishers Inc., Basel.

Wang H, Wang XQ, Xu XP and Lin GW (2010). Cytogenetic evolution correlates with poor prognosis in myelodysplastic syndrome. Cancer Genet. Cytogenet. 196: 159-166. 